\title{
Comment: "Cosmesis in patients with breast neoplasia submitted to the hypofractionated radiotherapy with of intensity-modulated beam"
}

1. Radiation oncologist at Albert Einstein Israeli Hospital in São Paulo, Brasil

The retrospective cohort study "Cosmesis in patients with breast neoplasia submitted to the hypofractionated radiotherapy with intensity-modulated beam"1, conducted by Dr. Fabiana Miranda at Hospital Sírio-Libanês, in São Paulo, shows the feasibility and the importance of maintaining radiation dose homogeneity delivered to breast cancer patients.

It is known that there is a greater probability of toxicities and complications for breast tissues that receive daily higher radiation doses due to heterogeneity, with the differences for biological effects being called "Double-Trouble" or "Triple-Trouble" effects ${ }^{2}$. For patients irradiated with hypofractionated schemes, dose coverage and dose homogeneity are even more important, as even small differences in radiation dose distribution may lead to great differences in the biological effect.

As far as I know, this is the first Brazilian publication about dose homogeneity that can be reached with the use of "Forward Planning" Intensity Modulated Radiation Therapy in the context of breast cancer, serving as basis and an example of care that must be used in order to reach not only good results in the tumor control, but also in breast cosmesis.

\section{REFERENCES}

1. Miranda FA, Vieira MTL, De Moraes FY, Marta GN, Carvalho HA, Hanna AS. Cosmesis in patients with breast neoplasia submitted to the hypofractionated radiotherapy with of intensity-modulated beam; Rev Assoc Med Bras 2018; 64 (11): 1023-1030

2. Lazzari G, Terlizzi A, Scaparti GDV, Perri F, et al. Predictive parameters in hypo fractionated whole-breast 3D conformal radiotherapy according to the Ontario Canadian trial. Onco Targets There 2017;10:1835-1842 